\title{
Corela
}

Cognition, représentation, langage

HS-35 | 2022

Anaphore et pronoms en anglais : convergences, différences et complémentarité de quelques approches linguistiques

\section{Perspective de la grammaire générative sur l'anaphore}

\section{Philip Miller}

\section{(2) OpenEdition \\ Journals}

Édition électronique

URL : https://journals.openedition.org/corela/14109

DOI : $10.4000 /$ corela. 14109

ISSN : 1638-573X

Éditeur

Cercle linguistique du Centre et de l'Ouest - CerLICO

\section{Référence électronique}

Philip Miller, « Perspective de la grammaire générative sur l'anaphore », Corela [En ligne], HS-35 | 2022, mis en ligne le 03 janvier 2022, consulté le 24 janvier 2022. URL : http://journals.openedition.org/ corela/14109; DOI : https://doi.org/10.4000/corela.14109

Ce document a été généré automatiquement le 24 janvier 2022.

\section{(c) (1)(2)}

Corela - cognition, représentation, langage est mis à disposition selon les termes de la licence Creative Commons Attribution - Pas d'Utilisation Commerciale - Partage dans les Mêmes Conditions 4.0 International. 


\title{
Perspective de la grammaire générative sur l'anaphore
}

\author{
Philip Miller
}

\section{Introduction}

1 Dans cette introduction, nous commencerons par clarifier la notion même de 'grammaire générative'. Ensuite, nous passerons rapidement en revue les contributions limitées de la grammaire générative chomskienne dans le domaine de l'anaphore. Puis, nous introduirons quelques concepts généraux qui nous seront utiles dans la discussion de l'anaphore dans une perspective générative plus large.

\subsection{Qu'est-ce que la grammaire générative?}

2 Chomsky 1957 définit la grammaire générative comme une théorie linguistique suffisamment explicite (c'est-à-dire suffisamment formalisée) pour qu'on puisse vérifier si une structure (syntaxique, phonologique, sémantique, morphologique, selon le cas) est définie comme bien formée ou non. Comme le modèle formel dont il disposait à l'époque (grammaires syntagmatiques avec règles de réécriture produisant des structures profondes et ensemble de transformations permettant de transformer celles-ci en structures de surface) est un mécanisme génératif, cela signifiait que la théorie devait être suffisamment explicite pour vérifier si une phrase est générée par le système ou non.

3 Depuis cette époque, le terme de 'grammaire générative' a acquis trois acceptions. D'une part, il désigne les théories syntaxiques successives défendues par Chomsky (théorie 'standard' : Chomsky 1965; théorie 'standard étendue': Chomsky 1972; théorie du 'gouvernement et du liage' : Chomsky 1981 ; théorie 'minimaliste' : Chomsky 1995), malgré le constat que ces théories deviennent de moins en moins 'génératives' au sens propre, puisqu'elles sont de moins en moins formalisées et donc de moins en moins prédictives. D'autre part, il s'utilise au sens originellement proposé par 
Chomsky, désignant toute théorie syntaxique (qu'elle soit transformationnelle et générative ou non) suffisamment explicite pour qu'on puisse déterminer si elle prédit ou non que des structures sont bien formées. Enfin, le terme s'utilise pour désigner des théories générales de la langue avec tous les composants et leurs interfaces, soit lorsqu'elles sont inspirées de la perspective chomskienne, soit lorsqu'elles se veulent suffisamment explicites. Dans cette voie, on parle aussi des autres composants de la grammaire (par exemple, phonologie générative et morphologie générative) à la fois dans le cas des théories inspirées par les travaux de Chomsky, et dans le cas de théories qui se veulent explicites et donc prédictives.

4 Le premier et le troisième emplois sont typiquement ceux de Chomsky et de ses disciples, mais il est aussi celui de nombreux linguistes qui ne se réclament pas de la grammaire générative, quelle que soit l'acception. Le second emploi est typiquement utilisé pour se désigner eux-mêmes par les linguistes qui s'attachent à produire des théories linguistiques suffisamment formalisées pour qu'on ait une idée relativement claire de ce qu'elles prédisent, même si ces théories n'ont généralement pas recours au mécanisme des transformations et ne sont pas génératives au sens strict, en ce sens qu'elles définissent explicitement la notion de structure bien formée par d'autres biais qu'un mécanisme qui génère celles-ci. En particulier, une grammaire peut être entendue comme un ensemble de principes de bonne formation qui permettent de vérifier si une structure est bien formée ou non. Parmi celles-ci on peut noter en particulier, la Grammaire Catégorielle (CG, voir, par exemple, sur une construction elliptique, Kubota et Levine 2017), la Grammaire Lexicale-Fonctionnelle (LFG, voir par exemple Bresnan 1982) , la Grammaire Syntagmatique Guidée par les Têtes (HPSG, voir Pollard et Sag 1994, Ginzburg et Miller 2019), la Grammaire de Construction (CXG, Goldberg 1995), etc.

5 Toutes les théories citées sont des modèles où la syntaxe joue un rôle central et est au coeur des recherches qui y sont menées. Néanmoins, la plupart d'entre elles ont des composants sémantiques, pragmatiques, morphologiques et phonologiques plus ou moins développés et ont l'ambition d'expliquer les interfaces de ceux-ci avec la syntaxe.

6 Pour ce qui est des recherches proprement phonologiques, morphologiques, sémantiques (en particulier la sémantique formelle, issue des travaux de Montague, cf., Dowty et al. 1981 et ses développements divers, par exemple, la DRT et SDRT, cf., Kamp et Reyle 1993) et pragmatiques (cf., Roberts 1996, Kadmon 2001, Ginzburg 2012), elles relèvent de la grammaire générative au sens large dans la mesure où elles sont formalisées et font des prédictions explicites sur la bonne formation des structures, quel que soit le composant.

7 Enfin, on entend parfois le terme de 'grammaire générative' utilisé improprement par certains collègues linguistes anglicistes pour désigner tout ce qui s'écrit en anglais et qui n'est pas manifestement de la grammaire descriptive, incluant en particulier toutes sortes d'auteurs qui ne s'identifieraient pas comme générativistes.

\subsection{L'anaphore en grammaire générative chomskienne}

8 Dans la grammaire générative chomskienne, en particulier suite à Chomsky 1981, le terme 'anaphore' désigne spécifiquement les réfléchis en -self et le réciproque each other. Il s'oppose à ce qu'il appelle les 'pronominaux' (pronoms personnels) et aux 
'expressions-R' (autres SN référentiels). Chomsky propose une théorie syntaxique dite du liage (voir Zribi-Hertz 1996 pour une introduction pédagogique en français ou Büring 2005) qui a pour but d'expliquer les possibilités d'occurrence et d'interprétation de ces éléments au niveau de la syntaxe de la phrase. Pour cette raison, les théories chomskiennes n'ont que peu de choses à apporter à l'étude de l'anaphore dans un texte. Néanmoins, on peut noter quelques phénomènes dans le texte de Robbins qui permettent d'illustrer les hypothèses de Chomsky et leurs limites. Ainsi, Chomsky propose que les 'anaphores' doivent être 'liées' par un antécédent 'dans leur domaine local' (ce qui peut se gloser en première approximation par 'dans la même proposition'). Par contre, les 'pronominaux' ne peuvent être liés dans leur domaine local. Ceci prédit une distribution complémentaire des 'anaphores' et des 'pronominaux', illustrée dans les deux exemples suivants du texte de Robbins :

(1) a. what we $e_{i}$ have to remind ourselves ${ }_{i},(1.69)$

b. * what we $\mathrm{i}_{\mathrm{i}}$ have to remind us $\mathrm{i}_{\mathrm{i}}$

(2) a. But how easy for me to tell him $\mathrm{i}_{\mathrm{i}}$ what he $\mathrm{i}_{\mathrm{i}}$ should do. (1. 96)

b. *But how easy for me to tell him ${ }_{i}\left[_{p}\right.$ what himself ${ }_{i}$ should do].

$9 \quad$ En (1a), ourselves ne peut être remplacé par us parce qu'il est lié par we dans la même proposition. A l'inverse, en (2a), avec les liens de coréférence indiqués par les indices, on ne peut remplacer he par himself parce qu'il n'est pas dans la même proposition que son antécédent him.

Néamoins, on sait que l'hypothèse de la distribution complémentaire entre réfléchis et non réfléchis ne tient pas dans tous les contextes et notre texte fournit un exemple pertinent de ce point de vue :

(3) I'm looking to see what is it that's shaping that person's $s_{i}$ ability to contribute, $\left[_{P}\right.$

$\mathrm{PRO}_{\mathrm{i}}$ to do ${ }_{\mathrm{SN}}$ something $\left[{ }_{\text {SPrép }}\right.$ beyond themselves $\left.\left.\mathrm{i}_{\mathrm{i}}\right]\right]$. (1. 43-44)

11 Dans cette phrase, le réfléchi peut se justifier par l'hypothèse que le verbe infinitif a un sujet qui est une proforme zéro ('PRO' dans le jargon chomskien), coréférente avec son antécédent person's, qui fournit un antécédent au réfléchi dans la proposition qui l'inclut (to do something beyond themselves). Cependant, on constate que nous sommes ici dans un cas où la distribution complémentaire ne tient pas, puisqu'il est parfaitement possible de remplacer themselves par them (parce que le pronom se trouve dans un SPrép complément du SN objet direct, voir Büring 2005, p.222sv. pour diverses propositions de traitement syntaxique). Ce type d'exemple a été discuté par Kuno 1987 et a reçu une analyse approfondie dans Zribi-Hertz 1989. Ces auteurs ont montré que dans ce type de cas, le choix induit un positionnement énonciatif, en particulier dans les textes narratifs, fondé sur le point de vue, le réfléchi permettant une focalisation au sens littéraire - par le point de vue du personnage. En (3), le choix du réfléchi suggère, selon ces théories, que c'est du point de vue de la 'person' que la contribution doit la dépasser et non du point de vue de l'énonciateur 'I'.

\subsection{Anaphore, proforme et ellipse}

Dans ce qui suit, nous n'adoptons pas cette terminologie chomskienne. Nous utilisons le terme 'anaphore' au sens classique. Suivant une tradition bien établie, nous faisons l'hypothèse que l'anaphore a deux réalisations, soit une proforme, soit une ellipse. Ce qui unit les deux cas est que l'interprétation est possible grâce à la récupération d'un antécédent dans le contexte discursif ou situationnel. Néanmoins, la distinction 
classique entre proforme et ellipse pose problème dès le moment où l'on accepte l'idée des morphèmes zéro. En effet tout phénomène d'ellipse apparente peut être analysé comme un cas de proforme zéro. Ainsi, dans l'exemple (3) ci-dessus, le sujet de l'infinitif était considéré comme étant une proforme zéro. On aurait pu tout aussi bien considérer que ce sujet est ellipsé. Pour prendre un exemple encore plus clair, dans la plupart des langues romanes, la position sujet des phrases finies n'est pas obligatoirement remplie. En portugais, par exemple, dans un contexte où l'on vient de dire que Pedro ne serait pas présent, on peut dire soit (4a), soit (4b) pour expliquer son absence ${ }^{1}$.

(4) "(Il) va à São Paulo."

a. Vai para São Paulo.

b. Ele vai para São Paulo.

Les chomskiens, entre autres, font l'hypothèse d'un pronom nul dans des configurations comme (4a), qu'ils appellent 'pro' ('petit pro', qui s'oppose au PRO vu en (3) par le fait qu'il apparait comme sujet de verbes finis), qui serait coindicé avec son antécédent (Pedro ${ }_{i}$, dans notre exemple) :

(5) pro $_{i}$ vai para São Paulo.

14 Mais d'autres théories proposent qu'il n'y a simplement pas de position syntaxique sujet dans ce type de phrase, et que l'interprétation anaphorique est obtenue par d'autres mécanismes (voir par exemple le cas de HPSG, résumé dans Ginzburg et Miller 2019 : 98 sv.).

Inversement, dans de nombreux cas, l'élément qui permet ou déclenche l'ellipse peut être réinterprété comme une proforme. Par exemple, Schachter 1977 propose d'interpréter l'auxiliaire préelliptique comme une proforme dans ce qu'on appelle traditionnellement l'ellipse post-auxiliaire (EPA, 'VP Ellipsis' en anglais). Une possibilité très similaire est celle proposée par Hardt 1993 selon qui l'élément anaphorique est une proforme zéro située derrière l'auxiliaire. On peut illustrer ces possibilités par les représentations suivantes pour l'exemple d'ellipse post-auxiliaire en (6b). (6)(i) représente l'hypothèse de Schachter, l'auxiliaire étant interprété comme une proforme qui reçoit son interprétation par combinaison de son sens propre (ici, par exemple, un may épistémique) avec le contenu récupéré à partir de l'antécédent, à savoir work today. La représentation (6)(ii) est celle de Hardt : la proforme nulle reçoit ce même contenu qui est combiné avec celui de may, cette fois syntaxiquement.

(6) a. - Sandy may work today.

(i)

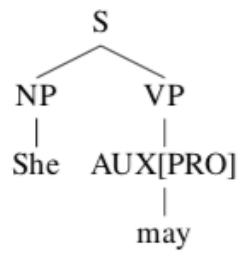

b. - She may.

(7) (ii)






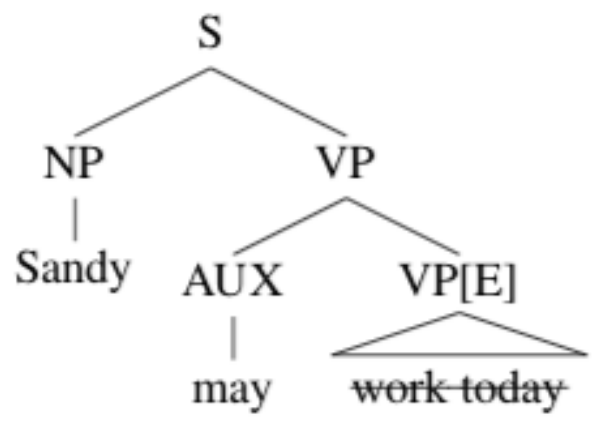

16 La représentation (7) illustre une troisième analyse où la structure attendue est présente en structure de surface mais n'est pas prononcée, ce qui est indiqué par le [E]. Cette dernière analyse est la plus commune dans les courants chomskiens actuels (voir par exemple Merchant 2001, Merchant 2013) et remplace l'idée que l'ellipse résulte d'un effacement syntaxique dans le passage entre structure profonde et structure de surface. Le choix entre ces possibilités (qui n'épuisent pas le détail de ce qu'on peut imaginer) n'est possible qu'en fonction d'hypothèses théoriques indépendantes, car toutes conduisent à la même interprétation et permettent donc de rendre compte des faits. Nous ne nous y attarderons pas ici, nous contentant de parler d'anaphore sans nous engager plus sur le fait de savoir s'il s'agit d'ellipse ou de proforme.

\subsection{Antécédent et déclencheur d'antécédent}

Cornish 1999 (qui ne s’identifie pas comme générativiste) propose de distinguer le déclencheur d'antécédent, à savoir le segment textuel qui permet de comprendre une anaphore, de l'antécédent au sens propre, à savoir la représentation conceptuelle qui permet l'interprétation de la proforme ou de l'ellipse. Cette distinction n'est pas nouvelle (elle apparaît par exemple dans Sag et Hankamer 1984, qui se situent pleinement dans le cadre de la grammaire générative), mais la terminologie de Cornish est particulièrement transparente. Dans (8), par exemple, le déclencheur d'antécédent qui permet d'interpréter l'ellipse post auxiliaire est lost one, mais l'antécédent est lose one $e^{2}$.

(8) I have never lost one in twenty-nine years. It doesn't mean that I won't some day. (1.39-40)

Bien que notre texte n'en compte pas, d'autres exemples présentent un écart plus frappant, considérons (9) et (10) :

(9) In a folded packet on the night table had been a pair of shining earrings.

- Are they your wife's?

She was trying one on, fastening it to her ear. [...]

-They were being repaired. I had to pick them up.

It was hard not to admire her, her bare neck, her aplomb.

-Can I borrow them? she asked.

-I can't. She knows I was supposed to pick them up. [=I can't lend them to you]. James Salter, "Platinum", Last Night.

(10) Mubarak's survival is impossible to predict and, even if he does [survive], his plan to make his son his heir apparent is now in serious jeopardy. $\left(\mathrm{COCA}^{3}\right)$ 
En (9) le déclencheur d'antécédent, I borrow them permet d'inférer l'antécédent lend them to you qui est son antonyme relationnel (cf. Lyons 1977), tandis qu'en (10), le déclencheur d'antécédent est le nom dérivé survival, qui permet de récupérer le verbe de base, cf. Miller et Hemforth 2014. Dans les cas de ce type, on parle d'anaphore avec discordance ('mismatch') entre l'antécédent et le déclencheur d'antécédent.

\section{Les premières approches génératives de l'anaphore}

\subsection{Substitution ou effacement sous identité syntaxique}

21 Dans la théorie dite 'standard', Chomsky 1965 distingue la structure profonde de la structure de surface (obtenue par transformations à partir de cette dernière) et suppose que la structure profonde est l'input de la sémantique tandis que la structure de surface est l'input de la phonologie. Dans cette perspective, il peut paraître naturel de faire l'hypothèse que les proformes sont obtenues par substitution de leur antécédent, présent en structure profonde. Cette opération, appelée 'pronominalisation' est illustrée dans l'exemple suivant, ou la version (b) indique la structure profonde supposée correspondre à la structure de surface en (a), dans laquelle la deuxième occurrence du SN antécédent est supprimée pour être remplacée par le pronom it. Cette opération est contrainte par le principe de 'récupérabilité des effacements' (recoverability of deletions, cf., Katz et Postal 1964, pp. 79-80 et Chomsky 1965, pp. 144-46), qui exige que l'effacement se fasse sous identité avec l'antécédent (la nature précise de l'identité en question étant sujette à débat, à la fois quant à savoir s'il s'agit d'identité de structure syntaxique ou de référence, et de savoir dans quelle mesure une certaine flexibilité doit être introduite).

(11) a. They're only going to get magnified by the very technology that's connecting

us, because it's making us intersect. (1. 29-30)

b. ... by the very technology that's connecting us, because the very technology

that's replacing us is making us intersect.

Ce type d'analyse remonte à Lees et Klima 1963 et a été soutenu jusque dans le début des années 70 par les tenants de la sémantique générative, qui se fonde sur l'hypothèse que la structure profonde est une structure sémantique (cf., p. ex., Ross 1969, Grinder et Postal 1971, Postal 1972).

Dans cette perspective, il est naturel d'étendre cette analyse à l'ellipse, la seule différence étant que l'effacement se fait sans substitution. Ceci est illustré en (12) dont la version (b) indique une fois de plus la structure profonde supposée, où le SV sousjacent à l'ellipse est effacé sous identité avec son antécédent lost one, souligné dans l'exemple.

(12) a. I have never lost one in twenty-nine years. It doesn't mean that I won't some day. (1. 39-40)

b. I have never lost one in twenty-nine years. It doesn't mean that I won't lose one some day.

Cependant, cette théorie rencontre plusieurs problèmes graves, qui ont conduit à son abandon par la très grande majorité des générativistes. D'une part, les transformations sont supposées avoir pour domaine d'application la phrase. Or, les anaphores ne sont souvent pas dans la même phrase que leur antécédent.

(13) because how did you learn language? You didn't learn it by just learning principles, (1. 6-7) 

est agrammatical. Seul une variante comme (16c), où la phrase antécédent est transformée en complétive en that, est recevable (la variante sans 'the fact' est grammaticale, mais difficilement contextualisable) $)^{4}$.

Ceci signifie qu'un cas comme (13) devrait être analysé par des mécanismes différents de ceux qui expliquent (11), ce qui semble peu explicatif.

Un deuxième argument similaire est fondé sur les emplois exophoriques des pronoms et des ellipses, illustrés en (14) :

(14) a. [Child with apple in hand, in front of monkey cage in zoo, monkey

approaching] Parent: Don't feed it. It might bite you.

b. [Child making annoying noise with toy.] Parent: Please stop doing that.

c. [Child about to touch hot stove.] Parent: Don't!

Dans la mesure où il n'y a pas d'antécédent, on voit mal comment ces énoncés pourraient être produits par substitution ou effacement sous identité. Il n'y a en effet aucun contenu linguistique permettant de vérifier celle-ci.

Le même type d'argument peut être construit lorsque l'antécédent est inféré à partir du contexte discursif. Considérons l'exemple de notre texte en (15) :

(15) [...] because how did you learn language? You didn't learn it by just learning principles, you got in it and you did it so often that it became real. (1. 6-7)

9 Dans cet exemple l'antécédent de do it n'est manifestement ni 'learn language', ni 'learn principles'. On infère plutôt, à partir du contexte discursif, un antécédent du type 'practiced' ou 'practiced using language' ou 'used language'. Il est important de noter qu'il s'agit d'une indétermination intrinsèque : il n'y a aucune raison de supposer que l'énonciateur ait l'une de ces formulations spécifiques à l'esprit plutôt qu'une autre, ni que le destinataire en reconstruise l'une plutôt que l'autre. L'indétermination qui en résulte ne pose pas de problèmes parce que les intentions référentielles de l'énonciateur sont suffisamment claires étant donné ses visées communicatives dans le contexte. Dans ce type de cas, qui n'est pas exophorique, le déclencheur d'antécédent n'est pas un segment textuel qu'on peut définir avec précision. On voit donc mal comment on pourrait appliquer le critère d'identité, qu'il soit interprété syntaxiquement ou sémantiquement.

Un troisième argument qui a pu être avancé contre la substitution et l'effacement est fondé sur des cas où la structure profonde supposée serait agrammaticale sans l'opération de substitution ou d'effacement. On peut illustrer ce cas par la phrase suivante de notre texte :

(16) a. You may not have the money, you may not have the Supreme Court, but that is not the defining factor. (1. 88)

b. *[...] but you may not have the money, you may not have the Supreme Court is not the defining factor.

c. [...] but (the fact) that you may not have the money or that you may not have the Supreme Court is not the defining factor.

Si on remplace le démonstratif that par son antécédent en (16a), on obtient (16b), qui Enfin, un dernier argument qui a été avancé contre cette idée est lié à des cas où l'antécédent d'une proforme contient une occurrence coréférente de cette même proforme. Ces phrases, connues sous le nom de 'Bach-Peters sentences' (cf., Bach 1970), conduisent donc à un paradoxe.

(17) I will reward $\left[_{\mathrm{SN}}\right.$ the man $\left[_{\mathrm{Rel}}\right.$ who shows he deserves the prize ] ] 
fait l'hypothèse que la relative Rel fait partie du SN dont la tête est $m a n^{5}$, ce $\mathrm{SN}$ qui réfère à l'entité $i$, contient une occurrence du pronom he, qui réfère à cette même entité. L'hypothèse de la substitution impliquerait que ce he aurait pour source une réplique de son antécédent, ce qui est illustré en (18) :

(18) I will reward $\left[_{S N}\right.$ the man $\left[_{P}\right.$ who shows $\left[_{S N}\right.$ the man $\left[_{P}\right.$ who shows he deserves the prize ] ] deserves the prize ] ]

On voit le paradoxe, puisque (18) ne peut être une structure profonde. Elle contient en effet une fois de plus le pronom he qui devrait avoir pour source une nouvelle substitution. On se trouve donc devant un cas de récursivité ('effet vache-qui-rit'), qui conduirait à accepter l'idée d'une structure profonde infinie. La découverte de cette classe d'exemples a été l'un des plus puissants arguments contre l'hypothèse de la substitution, puisqu'il fait apparaître un paradoxe pour cette analyse même dans le domaine de la phrase.

\subsection{Les théories interprétatives de l'anaphore}

En réaction aux difficultés qui viennent d'être illustrées, de nombreux générativistes ont proposé une approche interprétative des anaphores, à savoir l'idée selon laquelle toutes les anaphores, qu'il s'agisse de proformes ou d'ellipses, sont présentes telles quelles dès la structure profonde. L'antécédent est déterminé par un mécanisme sémantique interprétatif (cf., p. ex., Dougherty 1969 pour les pronoms et Jackendoff 1972 pour une extension à l'ensemble des cas d'anaphore).

Cependant, cette position posait problème à l'époque pour une série de cas d'ellipse où il semblait qu'il y ait une exigence d'identité syntaxique entre antécédent et segment ellipsé. Considérons l'exemple suivant de notre texte :

(19) because I know people that wanted to vote in your direction and didn't. (1. 101)

Si on fait l'hypothèse que l'antécédent 'vote in your direction' est inféré à partir du sens de la proposition précédente, sans aucune référence à sa structure syntaxique, on voit mal comment le mécanisme interprétatif permettant cette interprétation pourrait ne pas s'appliquer également à la variante (20), avec antécédents discordants, puisqu'elle implique clairement la même représentation sémantique 'voter en faveur de $\mathrm{X}$.

(20) ?because I know people that were inclined towards a vote in your direction and didn't.

38 A l'époque, ce type d'exemple était considéré comme agrammatical (les premiers exemples d'antécédents nominaux attestés, parallèles à (10) ci-dessus, sont donnés par Hardt 1993). Il semble en première analyse que ce qui rend (20) moins acceptable que (19), c'est que l'antécédent apparaît sous la forme du nom vote plutôt que du verbe. Nous reviendrons sur ce type de problème dans la dernière section de l'article, en particulier pour expliquer pourquoi l'antécédent nominal en (10) ne conduit pas à la même perte d'acceptabilité que (20).

\subsection{L'approche mixte de Hankamer et Sag 1976}

Face à cette situation, où les deux grandes approches de l'anaphore semblaient confrontées à des problèmes insurmontables, Hankamer et Sag ont proposé, dans leur article classique de 1976 'Deep and Surface Anaphora', une théorie mixte. En bref, ils

Corela, HS-35 | 2022 
proposaient que les deux théories sont correctes, mais qu'elles s'appliquent à des anaphores différentes.

Plus précisément, Hankamer et Sag proposent que les anaphores se divisent en deux sous-classes. D'une part, les anaphores profondes, qui sont présentes dès la structure profonde et ne sont donc pas obtenues par transformation. Elles reçoivent leur interprétation par un mécanisme interprétatif qui ne réfère pas à la syntaxe. D'autre part, les anaphores de surface (appelées ainsi puisque dans la structure profonde correspondante elles ne sont pas présentes) sont obtenues par des tranformations de substitution ou d'effacement sous identité syntaxique. Leur interprétation résulte donc d'un mécanisme syntaxique.

Cette hypothèse conduit à un ensemble de prédictions qui paraissaient très pertinentes, vu l'état de connaissance des données de l'époque. En effet, on prédit ainsi d'une part que les anaphores profondes permettent des emplois exophoriques tandis que les anaphores de surface ne les permettent pas et, d'autre part, que les anaphores profondes permettent des ajustements syntaxiques majeurs en cas de discordance entre déclencheur d'antécédent et antécédent tandis que les anaphores de surface ne les permettent pas. Néanmoins, l'élargissement des données prises en compte a conduit à remettre en cause ces hypothèses. Hankamer et Sag considèrent, par exemple, que l'EPA est une anaphore de surface tandis que les pronoms personnels et do it sont des anaphores profondes. Ces hypothèses permettent d'expliquer pourquoi les exemples $(14 a, b)$ et (15) ci-dessus sont bien formés, puisque les anaphores profondes sont supposées accepter des antécédents exophoriques et des antécédents non identiques. Par contre, des cas comme (9), (10) et (14c) constituent des contre-exemples, puisque une anaphore de surface, l'EPA, y apparaît dans ces mêmes conditions. Nous reviendrons sur ces cas dans la section 4 .

\section{Le choix des expressions anaphoriques en discours}

\subsection{La théorie de l'accessibilité d'Ariel}

Les limites manifestes des analyses chomskiennes de l'anaphore ont conduit à une série de travaux visant à dépasser le cadre syntaxique de la phrase afin de proposer une théorie plus générale du choix des expressions anaphoriques en discours. On peut poser la question du choix de façon très générale dans les termes suivants : quel type de $\mathrm{SN}$ utiliser pour accéder à un antécédent donné ? On peut distinguer trois grands courants (donnés dans leur ordre historique d'apparition) ${ }^{6}$ :

-La théorie du centrage : Grosz et al. 1995

-La théorie de l'accessibilité : Ariel 1990

-La théorie de la saillance : Gundel et al. 1993

Ces trois théories se basent sur des concepts apparentés (plus un antécédent est saillant, plus il est accessible) et font des prédictions relativement similaires. La discussion qui suit utilise les concepts d'Ariel, qui se fonde sur les deux hypothèses suivantes :

47 i. Les référents discursifs sont plus ou moins accessibles. 
ii. Les expressions anaphoriques sont spécialisées et permettent d'accéder à des référents plus ou moins accessibles.

Figure 1. L'échelle de marquage d'accessibilité

(Ariel $1990:$ 73, adapté)

Ariel concrétise ces hypothèses par son échelle de marquage d'accessibilité, donnée ici avec quelques simplifications dans la figure 1. Par exemple, l'énonciateur, en choisissant d'utiliser un pronom non accentué, indique au destinataire que le référent visé est très accessible. A l'inverse, le choix d'une description définie longue indique que le référent visé est très peu accessible.

Accessibilité Basse

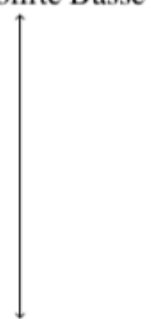

Accessibilité Haute

\author{
Description définie longue \\ Description définie courte \\ Démonstratif distal + modifieur \\ Démonstratif proximal + modifieur \\ Démonstratif distal $(+\mathrm{NP})$ \\ Démonstratif proximal $(+\mathrm{NP})$ \\ Pronom accentué \\ Pronom non accentué \\ Pronom nul
}

Pour échapper à la circularité, une telle analyse exige qu'on définisse les facteurs qui rendent une entité plus ou moins accessible indépendamment des anaphores qui permettent d'y référer. Selon Ariel, l'accessibilité dépend, d'une part, de facteurs qui sont intrinsèquement liés au type de référent: les entités de premier ordre (objets inanimés, êtres vivants) sont plus accessibles que les référents abstraits; les entités présentes situationnellement sont plus accessibles que celles qui ne le sont pas, etc. D'autre part, l'accessibilité des référents discursifs dépend de la structure du discours et, entre autres, des facteurs suivants :

50 - de la fonction discursive de l'antécédent (e.g., un référent qui était topique dans la phrase où il a été précédemment mentionné est plus accessible qu'un référent qui ne l'était pas);

51 - de la distance de l'antécédent (un antécédent plus distant est moins accessible) ;

52 - du fait qu'il soit dans la même unité textuelle ("paragraphe") ou non ;

53 - de son intégration syntaxique (un référent introduit dans une subordonnée sera moins accessible qu'un référent introduit dans une principale) ;

54 - de la compétition (s'il y a plusieurs référents potentiels en compétition avec le référent visé, il sera moins accessible).

Il est important de noter que ces facteurs ne sont pas indépendants.

\subsection{Application de la théorie de l'accessibilité au texte}

Nous allons maintenant illustrer comment cette théorie permet d'éclairer certains exemples du texte. Commençons par trois exemples de référents faiblement accessibles. On peut remarquer que dans ces trois cas, il n'y a pas à proprement parler d'anaphore, puisque les référents visés n'ont pas encore été mentionnés. La théorie d'Ariel est bien une théorie de la référence visant à expliquer le choix entre expressions

Corela, HS-35 | 2022 
référentielles, qu'elles soient anaphoriques ou non. Dans l'exemple (21), le référent visé a une accessibilité maximalement basse ${ }^{7}$.

(21) (1), 1.33. And that obsession is about what makes the difference in the quality of people's lives.

57 Il s'agit en effet d'un référent abstrait qui est en plus nouveau dans le discours. L'énonciateur choisit donc comme expression une description définie longue. De même dans (22), le référent visé a une accessibilité basse :

(22) (8), 1.56. what's the difference in somebody's life if you look at somebody like those people that you've given everything to?

Il s'agit une fois de plus d'un référent nouveau dans le discours. On s'attendrait donc à priori à avoir une description définie longue, comme en (21), et un tel choix aurait effectivement été possible dans ce contexte discursif. Le choix d'une forme du type 'démonstratif distal + modifieur' indique au destinataire que l'énonciateur pense qu'il s'agit d'un référent qui lui est familier même s'il n'a fait l'objet d'aucune mention préalable dans leurs discours partagés ( $\approx$ you know the kind of people I mean, voir Bowdle and Ward 1995). Dans l'exemple (23), le référent visé a une fois de plus une accessibilité très basse: il s'agit d'un référent abstrait (un événement) qui est nouveau dans le discours.

(23) (2), 1.35. I get the phone call when the athlete is burning down on national television, and they were ahead by five strokes and now they can't get back on the course.

Néanmoins on trouve ici comme expression une description définie courte, ce qui semble contradictoire avec ce qui a été dit de l'exemple (21). Notons cependant que si la phrase s'arrêtait après l'objet direct the phone call, l'identification du référent serait compromise. Ce sont en effet les informations contenues dans le reste de la phrase qui, sans faire partie du SN, permettent d'accéder à son antécédent. On a donc une situation qui est fonctionnellement similaire à celle de (21), mais où les informations additionnelles cruciales à l'identification n'apparaissent pas au sein du SN référentiel lui-même, mais sont postposées. On peut y voir une stratégie visant à intriguer le destinataire.

Tournons-nous à présent vers des emplois anaphoriques, en commençant par un exemple où le référent est très accessible.

(24) (3), 1.36. I get the phone call when the athlete is burning down on national television, and they were ahead by five strokes and now they can't get back on the course.

61 Le référent visé a une accessibilité maximalement élevée car il s'agit d'un référent humain qui vient d'être mentionné. Il est de plus sujet de la phrase dans laquelle il est introduit. L'énonciateur choisit donc un pronom personnel non accentué (avec le choix de they pour un référent singulier quantifié, qui permet d'éviter de préjuger du genre).

Considérons maintenant un cas où le référent a une accessibilité moyenne :

(25) (11), 1.108. If you don't have the money, but you're creative and determined enough, you find the way. So this is the ultimate resource, but this is not the story that people tell us.

63 L'antécédent visé est 'being creative and determined enough'. Celui-ci est récupéré à partir du déclencheur d'antécédent souligné. Il s'agit d'une entité abstraite (un état) et donc d'un référent inhéremment moins accessible qu'une entité de premier ordre. Le choix du démonstratif this permet d'indiquer au destinataire que l'énonciateur vise un 
référent moins accessible que ce qui serait visé par it (par exemple, 'the money', qui ne convient pas pragmatiquement néanmoins).

L'exemple suivant illustre un second cas de référent d'accessibilité moyenne, plus complexe :

(26) (7), 1.53. [...] there's two master lessons. One is: there's the science of achievement.

But the other lesson of life that is rarely mastered is the art of fulfillment. Because - you know, you up the ante, don't you? But when it comes to fulfillment - that's an art. And the reason is, it's about appreciation and it's about contribution.

Le contexte discursif oppose deux 'master lessons'. La première, 'the science of achievement', est développée 1.45-49. Ensuite, avec un changement de paragraphe, l'énonciateur introduit la deuxième leçon, 'the art of fulfillment', dans la première phrase du nouveau paragraphe, 1.50. Mais il l'abandonne aussitôt pour revenir à la première leçon, qu'il développe encore sur trois phrases 1.51-2. Il utilise ensuite la locution thématisante 'when it comes to' pour établir 'fulfillment' comme focus contrastif. En tant que tel, le SN qui l'exprime, that, doit recevoir un accent nucléaire. Deux facteurs (qui ne sont évidemment pas indépendants) conspirent donc pour imposer le choix de 'that' plutôt que de 'it'. La compétition entre deux antécédents possibles (qui rend les référents moins accessibles) et l'impossibilité de placer l'accent nucléaire sur 'it'. On notera incidemment que dans les deux propositions qui suivent, le même référent est repris par 'it'. Ceci est très typique. Ayant référé à une entité moyennement accessible par un démonstratif, l'énonciateur l'a promue au rang de référent très accessible, et les références additionnelles à la même entité qui suivent immédiatement se feront par un pronom non accentué8.

On peut aborder maintenant un cas plus particulier avec 'it'. Dans son emploi général d'anaphorique neutre, la théorie de l'accessibilité prédit qu'il aura pour antécédent le référent inanimé le plus accessible dans le contexte discursif. En effet, ce pronom ne contient aucune information proprement sémantique au-delà du fait que le référent n'est pas humain. Seules les contraintes sur l'accessibilité de son antécédent peuvent aider à repérer celui-ci. On trouve des occurrences de ce type par exemple 1. 7, 'learn it by just learning principles' (= language) ou 1. 47 , 'you take the invisible and make it visible' (= the invisible). Tournons-nous donc vers le cas suivant :

(27) (10), 1.104. [Discussion de l'importance de l'émotion en amont 1.90-94] But there was emotion that was there. How many know what I'm talking about here? Say, "Aye."

Audience: Aye.

So, emotion is it. If we get the right emotion, we can get ourselves to do anything. We can get through. If you're creative enough, playful enough, fun enough, can you get through to anybody, yes or no?

67 La première chose sur laquelle il faut insister est le fait qu'il s'agit d'un exemple tout à fait atypique. L'utilisation de 'it' comme attribut du sujet est très peu fréquente. Hors du slogan publicitaire 'Coke is it' (qui joue certainement sur l'étrangeté de la formulation), on trouve peu d'exemples de ce type dans un corpus comme le COCA 9 . On a donc ici une construction copulative spécificationnelle (cf. Higgins 1979) qui inverse l'ordre habituel de construction :

(28) (10') It is emotion. ( $\approx$ What I'm talking about is emotion.)

Le référent de 'it' est donné ici par son antécédent 'what I'm talking about' (1.102). Il s'agit donc d'un référent discursivement très accessible, ce qui explique que (10') serait 
une variante acceptable. Une motivation possible de l'ordre inversé inhabituel est la suite du texte qui élucide le 'what I'm talking about' auquel réfère it : 'What I am talking about is the fact that if we get the right emotion, we can get ourselves to do anything.'

Considérons à présent un cas de 'it' qui se prête à une double analyse :

(29) (9), 1.64. So, the question we've got to ask ourselves really is, what is it? What is it that shapes us?

Aucun référent plausible n'est très accessible dans le contexte discursif. Il ne s'agit pas non plus d'une occurrence de l'utilisation la plus habituelle de la locution 'What is it?' sans référent spécifique accessible pour 'it' ( $\approx$ 'What is going on?'). Il semble y avoir deux analyses possibles, conduisant à des interprétations similaires. La première consiste à dire que l'énonciateur viole de façon manifeste les contraintes sur l'emploi de 'it', qui, comme on l'a dit, renvoie à un antécédent maximalement accessible. Dans le cas général, ceci force le destinataire à accommoder un tel référent (c'est-à-dire à faire comme s'il était accessible, ce qui explique l'effet 'in medias res' de l'utilisation d'un pronom dans un incipit de roman, par exemple). Mais ici, il s'agit plutôt d'intriguer le destinataire, puisque la phrase est suivie immédiatement d'une séquence discursive qui éclaire ce dont il s'agit. La seconde analyse suppose que 'it' est ici le pronom impersonnel typique des clivées dans une clivée réduite qui, contrairement à l'habitude, précède la clivée complète, avec une fois de plus l'effet d'intriguer le destinataire. Comme les pronoms impersonnels ne sont pas référentiels, le choix de leur forme spécifique ne peut pas s'expliquer en termes d'accessibilité (mais diachroniquement, on constate que ce sont typiquement des pronoms de 3e personne indiquant un référent d'accessibilité maximale qui se grammaticalisent de la sorte).

Tournons-nous enfin vers l'exemple suivant :

(30) (6), 1.50-53. But the other lesson of life that is rarely mastered is the art of

fulfillment. [...] But when it comes to fulfillment - that's an art. Figure 2. Premiers résultats pour la requête when it [ $\left.\mathrm{v}^{\star}\right]$ to sur le COCA

On a affaire ici à une collocation, comme l'indiquent les données du COCA présentées

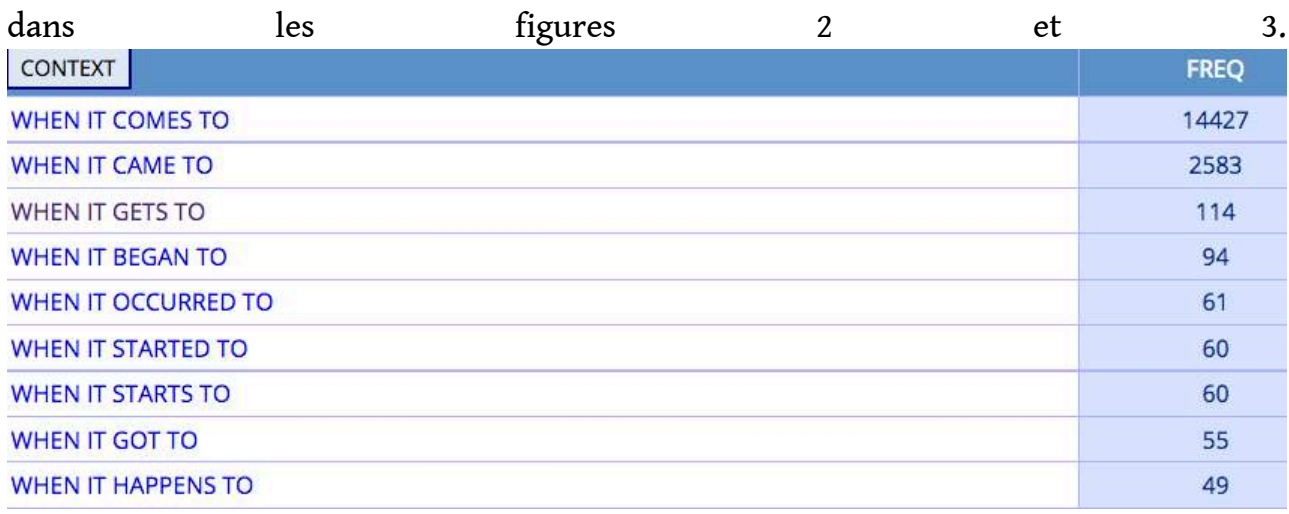

Figure 3. Premiers résultats pour la requête * it comes to sur le COCA

La figure 2 donne les résultats pour 'when it' suivi de n'importe quel verbe, suivi de 'to'. On voit que 'comes' dans 'When it V-s to' est environ 100 fois plus fréquent que le verbe 


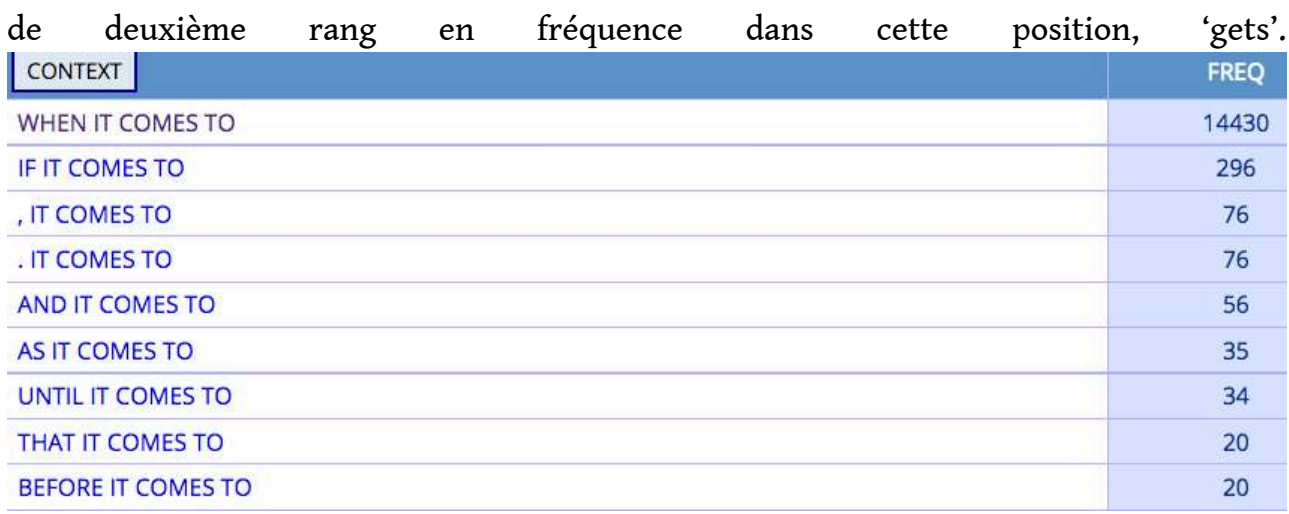

De même, la figure 3 donne les résultats pour n'importe quel mot ou ponctuation suivi de 'it comes to'. On constate une fois de plus que 'when' est beaucoup plus fréquent que la forme alternante qui suit, "if ${ }^{10}$.

Dans ce type de cas de figement, les alternances entre expressions référentielles en concurrence ne fonctionnent pas normalement et la théorie de l'accessibilité n'a donc pas à en rendre compte (du moins en synchronie ; comme pour les impersonnels, il se pourrait qu'on puisse expliquer diachroniquement pourquoi telle forme s'est prêtée au figement plutôt que telle autre).

\section{Les anaphores verbales}

\subsection{Introduction}

Dans cette section, nous nous pencherons sur les deux occurrences d'anaphores verbales soulignées dans le corpus commun ainsi que sur d'autres cas qui apparaissent dans le texte : le 'won't' est un cas d'Ellipse Post-Auxiliaire (EPA, 'Post-Auxiliary Ellipsis' (PAE), plus communément appelée 'Verb Phrase Ellipsis' (VPE) en anglais), tandis que le 'done it' est un cas d'Anaphore de SV (ASV, habituellement appelée 'Verb Phrase Anaphora' (VPA) en anglais).

(31) (4)-(5), 1.39-40. I'm very grateful to tell you I've never lost one in twenty-nine

years.

a. It doesn't mean I won't some day,

b. but I haven't done it.

Les théories interprétatives et transformationnelles n'ont aucun problème pour produire ces exemples ${ }^{11}$. Cependant, on notera qu'elles prédisent tout aussi bien la bonne formation des variantes suivantes, sans donner aucun indice sur ce qui conduirait à préférer (31a) et (31b) à (32a) et (32b) :

(32) I'm very grateful to tell you I've never lost one in twenty-nine years.

a. It doesn't mean I won't do it some day,

b. but I haven't.

Dans le contexte de la théorie de l'accessibilité, une hypothèse évidente serait que le choix entre ces formes (ainsi qu'entre celles-ci et do this, do that, do so) serait lié à l'accessibilité de l'antécédent. Il s'avère que l'extension de l'échelle d'accessibilité d'Ariel aux ASV do it, do this et do that conduit à de bonnes prédictions (sans doute parce que, comme le montre Flambard 2018, chapitre 3, ces formes sont compositionnelles, et héritent donc des propriétés d'accessibilité de it, this et that). 
Par exemple, on trouve une occurrence de do that dans le texte (1.65) :

(33) 1.65. And we live in a therapy culture. Most of us don't do that, ...

Le déclencheur d'antécédent est ici le nom therapy et l'antécédent inféré est 'undergo therapy ${ }^{12}$. La discordance entre le déclencheur d'antécédent et l'antécédent (le premier est nominal, le second verbal) rend le référent moins accessible, ce qui favorise do this/ that plutôt que do it, qui serait moins approprié ici.

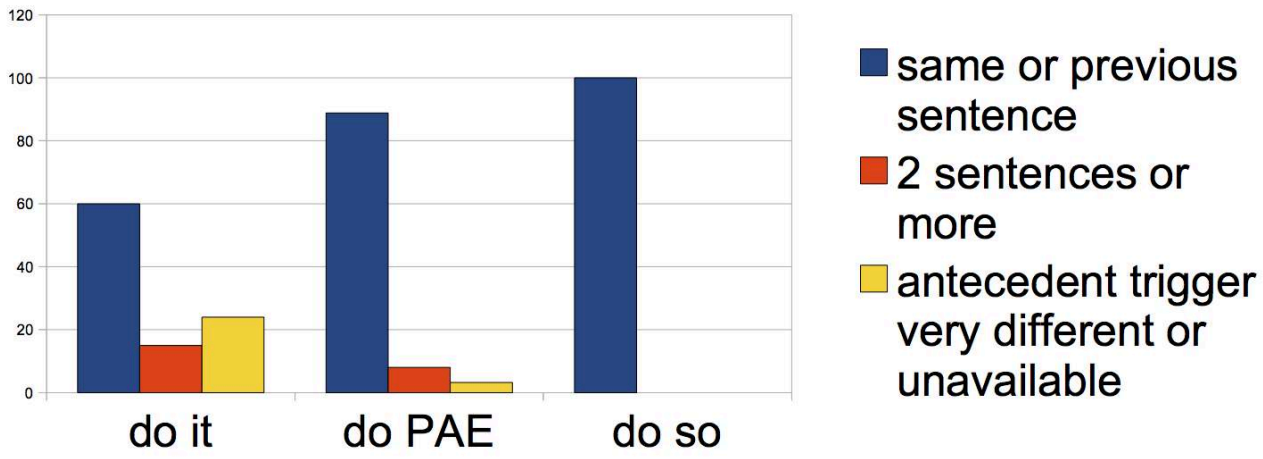

Pour ce qui est du choix entre l'EPA et les ASV 'do it' et 'do so', une petite étude menée sur un échantillon COCA (voir Miller 2011) suggère qu'il y a effectivement un effet de l'accessibilité (mesurée pour cette étude-d'une façon très opérationnelle mais un peu réductrice-en termes de distance en phrases entre l'anaphore et le déclencheur d'antécédent), comme l'indique la figure 4.

Figure 4. Distance en phrases entre anaphores verbales et déclencheur d'antécédents

Cependant il apparait que l'idée d'accessibilité ne suffit pas a expliquer les détails du choix entre anaphores verbales, ni les possibilités d'emploi de ces anaphores. Si nous revenons aux exemples (31a) et (32b) du texte, il semble difficile d'imaginer que le choix entre do it et l'EPA soit lié à l'accessibilité de l'antécédent puisqu'il est identique pour les deux formes.

\subsection{Conditions discursives sur le choix des anaphores verbales}

Quelles sont donc les conditions discursives sur l'emploi des anaphores verbales? C'est un sujet qui a été peu traité, en grammaire générative, comme ailleurs (voir cependant, dans la tradition énonciative, Guimier 1981 et Souesme 1985). Un certain nombre d'études ont tenté de proposer des conditions discursives sur l'EPA, visant à expliquer pourquoi certains cas d'EPA avec antécédents discordants sont acceptables tandis que d'autres ne le sont pas (voir en particulier Kehler 2002 et Kertz 2013). Cependant, les conditions discursives proposées sont très générales et s'appliquent de la même façon à tous les cas d'anaphore verbale. Malgré leur intérêt réel, ces propositions ne peuvent donc pas expliquer le conditionnement différentiel des anaphores individuelles, qu'il s'agisse de l'EPA, ou des ASV do it, do this, do that ou do so.

81 Dans une série de publications récentes, j'essaie, avec différents collaborateurs, d'apporter quelques éclaircissements sur cette question, et plus particulièrement sur la question du choix entre EPA et ASV, voir Miller 2011, Miller et Pullum 2014, Miller et Hemforth 2014, Miller 2014, Miller et al. 2019. Cette dernière publication propose la condition suivante, basée sur la notion de QUD (Question Under Discussion, voir Roberts 1996, Ginzburg 2012). L'idée est qu'à tout moment dans une conversation il y a 
une question en discussion. Une nouvelle phrase adresse normalement cette QUD ou une QUD inférable à partir d'elle, mais elle peut aller au-delà en fournissant des informations pertinentes pour une QUD non inférable, qui est ainsi introduite dans le contexte discursif. Ceci peut s'illustrer par les exemples suivants :

(34) a. Sue didn't write a song. She didn't.

b. Sue didn't write a song. Sam did.

d. Sue didn't write a song. Sam did it for her.

En (34) l'énonciation de la phrase initiale permet d'inférer différentes QUD, par exemple 'Did Sue write a song?' ou 'Who wrote a song?', qui sont respectivement adressées par les continuations données en (34a) (confirmation insistante, par exemple face à une expression d'incrédulité du destinataire) et (34b) (qui fournit un sujet focus contrastif). Par contre, la continuation donnée en (34c) ne se contente pas d'adresser les QUD inférables, mais va au-delà de celles-ci, pour donner en plus une réponse à la question du bénéficiaire de l'événement, 'For whom did Sam write a song?', cette dernière QUD n'étant pas inférable à partir de la phrase de départ.

Ces concepts étant mis en place, nous proposons la condition suivante sur le choix entre EPA et ASV :

(35) Si la QUD à laquelle répond la proposition anaphorique est inférable à partir de la proposition antécédent, l'EPA est préférée à do it. Dans le cas contraire (si la proposition anaphorique introduit une QUD qui va au-delà de ce qui est inférable à partir de la proposition antécédente), do it est préféré à l'EPA.

84 Ceci explique que les variantes en (36) sont moins acceptables que celles de $(34)^{13}$.

(36) a. Sue didn't write a song. \#Sam did it.

b. Sue didn't write a song. \#Sam did for her.

Ceci permet de revenir sur l'exemple suivant, déjà évoqué en (15) ci-dessus. Outre ce qui avait été dit, on constate la présence du circonstant non contrastif so often that it became real qui adresse une QUD non inférable à partir de la proposition antécédente, et favorise donc le choix de l'ASV do it, plutôt que de l'EPA did donnée en variante.

(37) 1.6-7. You didn't learn it by just learning principles, you got in it and you \{did it/\#did\} so often that it became real.

Nous avons corroboré empiriquement la validité de (35) par une expérience d'acceptabilité visant à vérifier que la présence d'un adjoint non contrastif diminuait l'acceptabilité de l'EPA tandis que son absence diminuait celle des ASV. A cette fin, nous avons construit 20 items expérimentaux du type (38), basés sur deux paramètres binaires, à savoir le choix entre EPA (do) et ASV (do it) et la présence ou non d'un circonstant non contrastif (Circ $+/-$ ), ce qui donne 4 conditions par item.

(38) a. Sue didn't write a song. Sam did. (EPA/Circ-)

b. Sue didn't write a song. Sam did it. (ASV/Circ-)

c. Sue didn't write a song. Sam did for her. (EPA/Circ+)

d. Sue didn't write a song. Sam did it for her. (ASV/Circ+) 


\section{progress}

Emily didn't compose a melody.

Edward did.

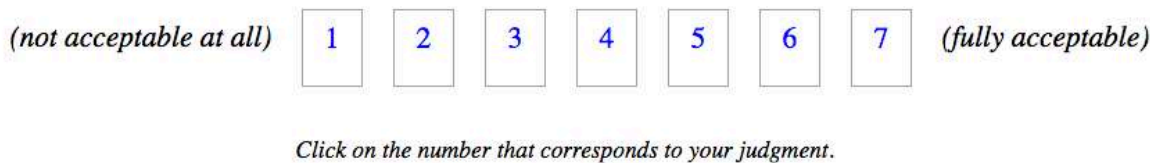

Ces 20 items ont été répartis en 4 listes (afin que chaque sujet expérimental ne voie qu'une seule des 4 conditions de chaque item; les sujets voyaient donc 5 items dans la condition EPA/Circ-, 5 dans la condition ASV/Circ-, etc.). Dans chacune de ces 4 listes, 16 distracteurs (phrases à juger venant d'une autre expérience, n'ayant rien de commun avec celles qui nous intéressent) ont été ajoutés, et chaque liste a été ordonnée aléatoirement. 48 sujets expérimentaux (donc 12 par liste, chaque phrase expérimentale recevant ainsi 12 jugements), recrutés sur Amazon Mechanical Turk (https://www.mturk.com/) ont jugé l'acceptabilité (expliquée en terme de 'naturalness') de la 2e phrase (soulignée) dans le contexte de la 1e, sur une échelle de 1 à 7. Un exemple de stimulus est donné dans la figure 5.

Figure 5. Exemple de stimulus

87 Les données d'un sujet ont été éliminées parce qu'il a dit, dans le questionnaire initial permettant d'identifier différentes propriétés pertinentes des sujets, qu'il était bilingue. Chaque stimulus était suivi d'une question polaire pour assurer que les sujets 
étaient attentifs. Nous avons utilisé la plateforme Ibex Farm (Drummond 2014) pour réaliser l'expérience. Un résumé des résultats est donné dans la figure $6^{14}$.

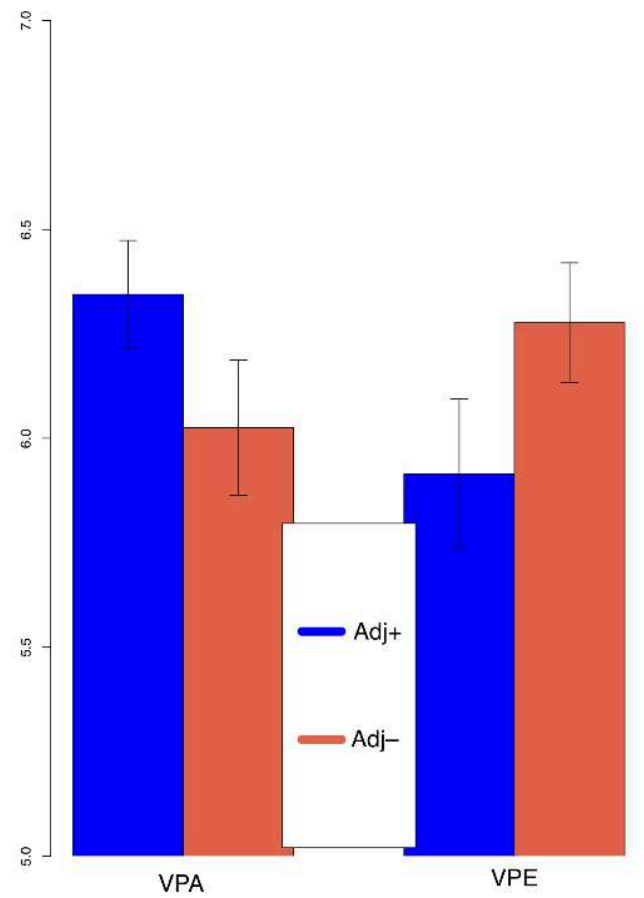

Figure 6. Acceptabilité des 4 conditions

En bref, le choix entre Circ+ et Circ- en tant que tel ne donne lieu à aucun effet significatif, pas plus que celui entre l'EPA et l'ASV (c-à-d que dans l'ensemble, les phrases avec circonstant ne sont pas jugées meilleures ou moins bonnes que celles sans circonstants et que celles avec EPA ne sont jugées ni meilleures ni moins bonnes que celles avec ASV). Par contre, on constate une interaction très significative $(p<0.0027)$ entre les deux facteurs, ce qui corrobore la condition (35) : la présence d'un circonstant non contrastif favorise l'ASV tandis que son absence favorise l'EPA.

Revenons un instant aux exemples (4) et (5) du texte :

(39) (4)-(5), 1.39-40. I'm very grateful to tell you I've never lost one in twenty-nine

years.

a. It doesn't mean I won't some day,

b. but I haven't done it.

On pourrait penser que la présence du circonstant temporel some day dans (39a) va à l'encontre de ce qui vient d'être proposé. Mais ce n'est pas le cas, parce qu'il ne s'agit justement pas d'un circonstant non contrastif : en effet, some day s'oppose à never dans la phrase précédente. La présence de never dans la proposition antécédent fait que la question de l'occurrence de l'événement à un moment ou à un autre fait bien partie des QUD inférables. L'EPA est donc parfaitement naturelle dans ce contexte. Nous n'avons pas, par contre, à l'heure actuelle, d'explication du choix de l'ASV haven't done it, plutôt que de l'EPA haven't dans (39b). Clairement il reste beaucoup à faire pour rendre compte de l'ensemble des facteurs pertinents dans ces alternances.

91 Néanmoins, avant de conclure, nous voudrions revenir un instant sur la différence entre les exemples (10) et (33), que nous citons à nouveau ici : 
(40) Mubarak's survival is impossible to predict and, even if he does [survive], his plan to make his son his heir apparent is now in serious jeopardy. (COCA)

(41) 1.65. And we live in a therapy culture. Most of us don't do that, ... la discordance catégorielle entre antécédent et déclencheur d'antécédent? Comme le montrent Miller et Hemforth 2014, survival est un nom d'un type particulier (qu'ils appellent 'noms polaires') qui, dans certains contextes introduisant des interrogatives indirectes, peuvent véhiculer une signification proche de celle d'une interrogative polaire. En effet, (40) se paraphrase facilement par 'Whether or not Mubarak will survive is impossible to predict and, even if he does, his plan ...'. On voit donc ici que la proposition antécédent rend accessible la QUD ‘Will Mubarak survive or not?' et la phrase elliptique adresse la question en choisissant l'une des deux réponses. (35) prédit donc que l'EPA sera préférée à une ASV, ce qui est effectivement le cas. Par contre, aucune paraphrase de ce type n'est possible pour (41). On voit donc que la sémantique particulière du nom survival est centrale dans l'acceptabilité de l'EPA avec antécédent nominal illustrée dans ce type de cas.

\section{Conclusion}

Nous avons, dans cet article, proposé un parcours à travers ce qui a pu être dit de l'anaphore dans la grammaire générative, en partant d'une acception étroite (école de Chomsky) et en élargissant à différents courants issus de celle-ci. Les analyses centrées sur la syntaxe permettent de résoudre certaines questions relativement simples (les cas canoniques d'emplois des réfléchis, par exemple), mais elles montrent très vite leur limites : l'anaphore est manifestement un phénomène essentiellement discursif. Mais pour ceux qui ont adopté la consigne initiale de Chomsky, visant à être le plus explicite possible dans leurs analyses, les limites de l'approche syntaxique dans ce genre de cas ont conduit à essayer de formaliser les aspects pertinents du discours (voir en particulier Ginzburg 2012) afin de pouvoir faire des prédictions explicites de bonne formation.

\section{BIBLIOGRAPHIE}

Ariel, M. (1990). Accessing Noun Phrase Antecedents. London: Routledge.

Bach, E. (1970). Problominalization. Linguistic Inquiry 1, 121-122.

Bowdle, B.F. \& Ward, G. (1995). Generic demonstratives. In Annual Meeting of the Berkeley Linguistics Society, 32-43. Berkeley, CA.

Bresnan, J. (ed.) (1982). The Mental Representation of Grammatical Relations. Cambridge, MA: MIT Press.

Büring, D. (2005). Binding Theory. Cambridge: Cambridge University Press.

Corela, HS-35 | 2022 
Chomsky, N. (1957). Syntactic Structures. The Hague : Mouton.

Chomsky, N. (1965). Aspects of the Theory of Syntax. Cambridge, MA: MIT Press.

Chomsky, N. (1972). Studies on Semantics in Generative Grammar. The Hague: Mouton.

Chomsky, N. (1981). Lectures on Government and Binding. Dordrecht: Foris.

Chomsky, N. (1995). The Minimalist Program. Cambridge, MA: MIT Press.

Cornish, F. (1999). Anaphora, Discourse, and Understanding: Evidence from French and English. Oxford: Oxford University Press.

Culicover, P.W. \& Jackendoff, R.S. (2005). Simpler Syntax. Oxford : Oxford University Press.

Davies, M. (2008-). The Corpus of Contemporary American English: 450 million words, 1990-present. http ://corpus.byu.edu/coca/.

Dougherty, R.C. (1969). An interpretive theory of pronominal reference. Foundations of Language 5(4), 488-519.

Dowty, D.D., Wall, R.E. \& Peters, S. (1981). Introduction to Montague Semantics. Dordrecht: Reidel.

Drummond, A. (2014). Ibex Farm. http ://spellout.net/latest ibex manual.pdf.

Flambard, G. (2018). English VP Anaphors: do it, do this, do that. Thèse de doctorat. Paris : Université Paris Diderot.

Ginzburg, J. (2012). The Interactive Stance. Oxford: Oxford University Press.

Ginzburg, J. \& Miller, P. (2019). Ellipsis in Head-Driven Phrase Structure Grammar. In Craenenbroek, J. van \& Temmerman, T. (eds.) The Oxford Handbook of Ellipsis, 75-121. Oxford: Oxford University Press.

Goldberg, A.E. (1995). Constructions: A Construction Grammar Approach to Argument Structure. Chicago: University of Chicago Press.

Grinder, J. \& Postal, P.M. (1971). Missing antecedents. Linguistic Inquiry 2, 269-312.

Grosz, B., Joshi, A. \& Weinstein, S. (1995). Centering: A framework for mode ling the local coherence of discourse. Computational Linguistics 21, 203-225.

Guimier, C. (1981). Sur la substitition verbale en anglais. Modèles Linguistiques 3(1), 135-161. Gundel, J., Hedberg, N. \& Zacharski, R. (1993). Cognitive status and the form of referring expressions in discourse. Language 69(2), 274-307.

Hankamer, J. \& Sag, I.A. (1976). Deep and surface anaphora. Linguistic Inquiry 7, 391- 426.

Hardt, D. (1993). Verb Phrase Ellipsis: Form, Meaning, and Processing. Ph.D. thesis, University of Pennsylvania. Distributed as IRCS Report 93-23.

Higgins, R.F. (1979). The Pseudo-cleft Construction in English. Outstanding Dissertations in Linguistics. New York: Garland.

Jackendoff, R.S. (1972). Semantic Interpretation in Generative Grammar. Cambridge, MA: MIT Press.

Kadmon, N. (2001). Formal Pragmatics: Semantics, Pragmatics, Presupposition and Focus. Oxford: Blackwell.

Kamp, H. \& Reyle, U. (1993). From Discourse to Logic. Dordercht: Kluwer.

Katz, J.J. \& Postal, P.M. (1964). An Integrated Theory of Linguistic Descriptions. Cambridge, MA: MIT Press. 
Kehler, A. (2002). Coherence, Reference, and the Theory of Grammar. Stanford: CSLI Publications.

Kertz, L. (2013). Verb phrase ellipsis: The view from information structure. Language 89, 390-428.

Kubota, Y. \& Levine, R. (2017). Pseudogapping as pseudo-VP ellipsis. Linguistic Inquiry 48(2), 213257.

Kuno, S. (1987). Functional Syntax: Anaphora, Discourse, and Empathy. Chicago: University of Chicago Press.

Lees, R.B. \& Klima, E.S. (1963). Rules for English pronominalization. Language 39, 17-28.

Lyons, J. (1977). Semantics. Cambridge: Cambridge University Press.

Merchant, J. (2001). The Syntax of Silence: Sluicing, Islands, and the Theory of Ellipsis. Oxford: Oxford University Press.

Merchant, J. (2013). Voice and ellipsis. Linguistic Inquiry 44, 77-108.

Miller, P. (2011). The choice between verbal anaphors in discourse. In Hendrickx, I., Lalitha Devi, S., Branco, A. \& Mitkov, R. (eds.) Anaphora Processing and Applications: 8th Discourse Anaphora and Anaphor Resolution Colloquium, DAARC 2011, Volume 7099 of Lecture Notes in Artificial Intelligence, 82-95. Berlin: Springer.

Miller, P. 2014. Les compléments orphelins dans les ellipses et anaphores verbales en anglais. In Girard, G. (dir.) Autour du verbe anglais, 45-57. Paris : Presses de la Sorbonne Nouvelle.

Miller, P. \& Hemforth, B. (2014). Verb phrase ellipsis with nominal antecedents. Ms. Université Paris Diderot.

Miller, P., Hemforth, B., Amsili, P. \& Flambard, G. (2019). Missing antecedents found. Soumis à publication.

Miller, P. \& Pullum, G.K. (2014). Exophoric VP ellipsis. In Hofmeister, P. \& Norcliffe, E. (eds.) The Core and the Periphery: Data-driven Perspectives on Syntax Inspired by Ivan A. Sag, 5-32. Stanford, CA: CSLI Publications.

Pollard, C. \& Sag, I.A. (1994). Head-Driven Phrase Structure Grammar. Chicago: Chicago University Press.

Postal, P. (1972). Some further limitations of interpretive theories of anaphora. Linguistic Inquiry 3, 349-371.

Roberts, C. (1996). Information structure in discourse: Towards an integrated formal theory of pragmatics. In Yoon, J.-H. \& Kathol, A. (eds.) Ohio State University Working Papers in Linguistics, vol. 49, 91-136. Columbus, OH: Ohio State University.

Ross, J.R. (1969). On the cyclic nature of pronominalization. In Reibel, D. \& Schane, S. (eds.)

Modern Studies in English, 187-200. Englewood Cliffs, NJ: Prentice-Hall.

Sag, I.A. \& Hankamer, J. (1984). Towards a theory of anaphoric processing. Linguistics and Philosophy 7, 325-345.

Schachter, P. (1977). Does she or doesn't she? Linguistic Inquiry 8, 763-767.

Soares, E.C, Miller, P. \& Hemforth, B. (2019). The effect of verbal agreement marking on the use of null and overt subjects - a quantitative study of first person singular in Brazilian Portuguese. Forum Linguístico 16.1, 3579-3600.

Souesme, J.-C. (1985). Do Something et ses diverses réalisations en anglais contemporain. Thèse de doctorat. Paris : Université Paris 7. 
Zribi-Hertz, A. (1989). Anaphor binding and narrative point of view: English reflexive pronouns and discourse. Language 65, 695-727.

Zribi-Hertz, A. (1996). L'anaphore et les pronoms : une introduction à la syntaxe générative. Villeneuve d'Ascq : Presses Universitaires du Septentrion.

\section{NOTES}

1. Ceci est surtout vrai en portugais européen, où le choix se ferait selon les visées discursives, (4b) se prêtant par exemple à une interprétation contrastive. En portugais du Brésil, la variante (4a) est beaucoup moins naturelle, voir par exemple Soares et al. 2019 pour des références.

2. Pour clarifier l'interprétation visée des exemples nous soulignons le déclencheur d'antécédent et double soulignons l'élément anaphorique.

3. Le COCA (Corpus of Contemporary American English, voir Davies 2008) est un corpus étiqueté de 560 millions de mots, accessible en ligne.

4. Cet argument ne serait pas considéré probant par la majorité des générativistes, car ils ne font pas l'hypothèse que la structure profonde doive correspondre directement à une structure bien formée.

5. Ceci est justifié par le test de substition qui montre qu'on ne peut pas remplacer the man par him en laissant derrière la relative (*him that shows he deserves the prize) et donc que dans (17), the man ne constitue pas un SN.

6. Les auteurs mentionnés n'identifieraient sans doute pas leurs travaux comme relevant de la 'grammaire générative' même dans une acception large. Néanmoins, leurs travaux résultent au moins en partie d'une réaction aux limites manifestes de la théorie de Chomsky dans le domaine de l'anaphore, et ils ont influencé la théorie générative au sens large.

7. Pour renvoyer aux exemples soulignés et numérotés par les éditeurs du volume dans le corpus commun, nous répétons leur numéro et le numéro de ligne en début d'exemple.

8. A l'inverse, on peut noter que dans (25) le démonstratif souligné que nous avons commenté est suivi par une deuxième occurrence formellement identique ('but this is not the story ...'). Le choix du démonstratif, plutôt que de it, permet d'indiquer que le référent visé n'est pas le même que celui auquel il vient d'être fait référence par le premier this (sans quoi c'est it qui aurait été utilisé), mais un nouveau référent abstrait.

9. La requête . $\left[\mathrm{n}^{*}\right]$ is it . (à savoir un point suivi de n'importe quel nom, suivi de 'is it', suivi d'un point) ne donne que 3 exemples pertinents avec un indénombrable. En élargissant la requête par suppression du point initial, on obtient 133 occurrences dont la très grande majorité sont non pertinents.

10. Dans ces deux cas, il est important d'établir la fréquence absolue des termes dans le corpus pour vérifier la pertinence des différences indiquées. En particulier, la forme 'comes' a 117.360 occurrences et 'gets' 78.359. De même, 'when' a 1.349 .336 occurrences et 'if 1.312.546. Les grandes différences constatées ne sont donc pas une simple conséquence d'une grande asymétrie de fréquence entre les termes en général. 
11. Notons qu'il est parfois dit que do it/this/that exigent un antécédent agentif (cf. Culicover et Jackendoff 2005, p.284), ce qui est contredit par l'exemple (31) (et de nombreux autres exemples attestés, voir en particulier Flambard 2018, chapitre 4).

12. Une seconde interprétation est possible où 'do' serait le verbe plein habituel et 'that' serait un démonstratif avec l'antécédent nominal 'therapy', mais dans ce cas, l'interprétation est plutôt l'activité du thérapeute que celle du patient, ce qui ne semble pas être le sens du texte.

13. Les jugements sont donnés en supposant une intonation neutre. Une intonation marquée peut modifier les choses, par exemple si 'Sam did' et 'for her' en (36b) forment deux groupes intonatifs séparés, cela devient beaucoup plus acceptable.

14. Les étiquettes de la figure correspondent aux abréviations anglaises : VPA $=$ ASV, VPE $=E P A$, Adj+/- = Circ+/-.

\section{RÉSUMÉS}

Cet article présente l'analyse de l'anaphore en grammaire générative. Après un rappel des différentes théories désignée par ce terme, il résume l'histoire du traitement de l'anaphore en grammaire générative. Il introduit ensuite la théorie de l'accessibilité d'Ariel et montre comment elle permet de rendre compte de certains choix d'expressions anaphoriques fournies par le texte. La section finale présente les travaux récents de l'auteur sur le choix entre anaphores verbales (l'Ellipse Post-Auxiliaire et les Anaphores de Syntagme Verbal do it, do this, do that). Il est montré que l'accessibilité permet de rendre compte de certains aspects du choix, mais qu'elle n'est pas suffisante. De nouvelles contraintes sur le choix sont proposées en termes de la notion de Question Sous Discussion, et sont corroborées par des données expérimentales.

This paper presents the treatment of anaphora in generative grammar. After a broad review of of the different theories covered under the term, it provides an overview of the history of the treatment of anaphora in generative grammar. It then discusses Ariel's theory of accessibility and shows how it can account for the choice of some of the anaphoric expressions provided in the text. In a final section, the paper presents the author's recent work on the choice between verbal anaphors (Verb Phrase Ellipsis and the Verb Phrase Anaphors do it, do this, do that). It is shown that accessibility can account for some aspects of the choice but is not sufficient. Further constraints on the choice are proposed in terms of the notion of Question Under Discussion (QUD), for which experimental evidence is adduced.

\section{INDEX}

Mots-clés : Ellipse, Anaphore, Ellipse Post-Auxiliaire, Question Sous Discussion, Antécédent, Grammaire Générative.

Keywords : Ellipsis, Anaphora, VP-Ellipsis, Question Under Discussion, Antecedent, Generative Grammar 
AUTEUR

PHILIP MILLER

Université Paris Diderot 